\title{
Centaurus A: Hard X-ray and high-energy gamma-ray light curve correlation
}

\section{Isak D. Davids*}

North-West University, Centre for Space Research, Private Bag X6001, Potchefstroom 2520,

South Africa, and

University of Namibia, Private Bag 13301, Department of Physics, Windhoek, Namibia, 12010

E-mail: isak.davids@gmail.com

\section{Markus Böttcher}

North-West University, Centre for Space Research, Private Bag X6001, Potchefstroom 2520,

South Africa

E-mail: Markus.Bottcher@nwu.ac.za

\section{Michael Backes}

University of Namibia, Private Bag 13301, Department of Physics, Windhoek, Namibia, 12010

and

North-West University, Centre for Space Research, Private Bag X6001, Potchefstroom 2520,

South Africa E-mail: mbackes @unam.na

\begin{abstract}
Centaurus A (Cen A), powered by a 55 million solar mass supermassive black hole, has been intensively monitored in all accessible wavelength ranges of the electromagnetic spectrum. However, its gamma-ray flux, both in the H.E.S.S. very-high and the Fermi-LAT high-energy gammaray regimes, is relatively faint, hampering light curve analyses. Yet, the extensive long-term light curve data from Fermi-LAT and Swift-BAT (hard X-rays) allows for cross-correlation studies. We find a hint that X-ray emission from Cen A precedes the gamma rays by 25 days. If this lag is real and related to a gamma-gamma absorption effect in the broad-line region (BLR) around the central source, then we can constrain the size of the BLR using light-travel time arguments. These are first results of extended light curve correlation studies between high-energy gamma rays and $\mathrm{X}$-rays from Cen A.
\end{abstract}

5th Annual Conference on High Energy Astrophysics in Southern Africa

4-6 October 2017

University of the Witwatersrand (Wits), South Africa

* Speaker. 


\section{Abbreviations}

The following abbreviations are frequently used in this manuscript:

$\begin{array}{ll}\text { BAT } & \text { Burst Alert Telescope (on-board Neil Gehrels Swift Observatory) } \\ \text { BLR } & \text { Broad Line Region } \\ \text { DCF } & \text { Discrete Correlation Function } \\ \text { HE } & \text { High-Energy (gamma rays of energy } \sim 0.1-100 \mathrm{GeV} \text { ) } \\ \text { H.E.S.S. } & \text { High-Energy Stereoscopic System } \\ \text { IACT } & \text { Imaging Atmospheric Cherenkov Telescope } \\ \text { LAT } & \text { Large Area Telescope (on-board Fermi satellite) } \\ \text { RG } & \text { Radio Galaxy } \\ \text { SED } & \text { Spectral Energy Distribution } \\ \text { VHE } & \text { Very High-Energy (gamma rays of energy } \gtrsim 100 \mathrm{GeV} \text { ) }\end{array}$




\section{Introduction}

Radio Galaxies (RGs) belong to the type of active galactic nuclei (AGN) for which the jet viewing angle with respect to our line of sight is relatively large in contrast to blazars for which this angle is small. A well-studied near-by radio galaxy is Centaurus A (Cen A). These sources are in general featuring a broadband (from radio to $\gamma$-rays) emission spectrum which is primarily attributed to non-thermal radiation. Two distinct peaks can be seen in such a spectral energy distribution (SED) where the lower energy peak (extending from radio to X-rays) is traditionally accepted as being due to synchrotron emission from relativistic electrons from the jet. The second hump (from X-rays to $\gamma$-rays) in the SED is attributed to inverse-Compton upscattering of a soft target photon field by the same relativistic jet electrons.

Amongst the basic components of the structure of a typical AGN, are the fast-moving high-density clouds surrounding the central engine (but external to the jet) and located closer to the central super-massive black hole (SMBH) than the optically obscuring dust torus. Due to the broad emission lines from this region, observed in the optical, these components are referred to as the broad line regions (BLRs) of the AGN. The location of the BLR can be measured by the reverberation method employing the light travel time in conjunction with the observable temporal lag in different energy band emission. The intense BLR radiation field is likely to be an efficient target photon field for $\gamma \gamma$-absorption producing electron-positron $\left(e^{-} e^{+}\right)$pairs [1,2]. On the other hand, most of the X-ray emission from AGN arises from the inner regions around the SMBH (see e.g. [3]).

In this work, we report results from a discrete cross-correlation of light curves as applied to SwiftBAT X-ray and Fermi-LAT $\gamma$-ray long-term observations of the nearest radio galaxy, Cen A.

\section{Centaurus A}

The massive elliptical galaxy NGC 5128 (discovered in 1826), is the closest radio galaxy (often referred to as Cen A), at a distance of $3.4 \mathrm{Mpc}$ [4]. This source is centrally powered by a SMBH of mass $5.5 \times 10^{7} \mathrm{M}_{\odot}$ (see e.g. [5, 6]). The radio lobes of Cen A subtend $\sim 10^{\circ}$ on the sky, due to both their gigantic sizes and the proximity of the source to Earth. Cen A is a Fanaroff-Riley Type I radio galaxy [7]. The source has been observed from radio to very-high energy (VHE; $E \gtrsim 100 \mathrm{GeV}$ ) $\gamma$-rays, and is an ideal target for studying radio lobes and relativistic outflows.

Numerous extensive studies have so far been carried out on Cen A, aiming at characterizing the SED of the source. A break in the $\gamma$-ray spectrum of the source above $2.4 \mathrm{GeV}$ has been established [8]. This break is not well modeled using a leptonic synchrotron self-Compton model. Recent results, from a joint initiative by H.E.S.S. and Fermi-LAT [9], show that the VHE data points are consistently above the high-energy (HE; $E \sim 0.1-100 \mathrm{GeV}$ ) power-law extrapolation of the $\gamma$-ray spectrum measured below the break. There is clear evidence for a second spectral component in VHE $\gamma$-rays in the SED.

As part of the aforementioned studies, various theoretical models with different emission mech- 
anisms and particle populations are usually employed to describe the SED of Cen A, as shown in Figure 1.

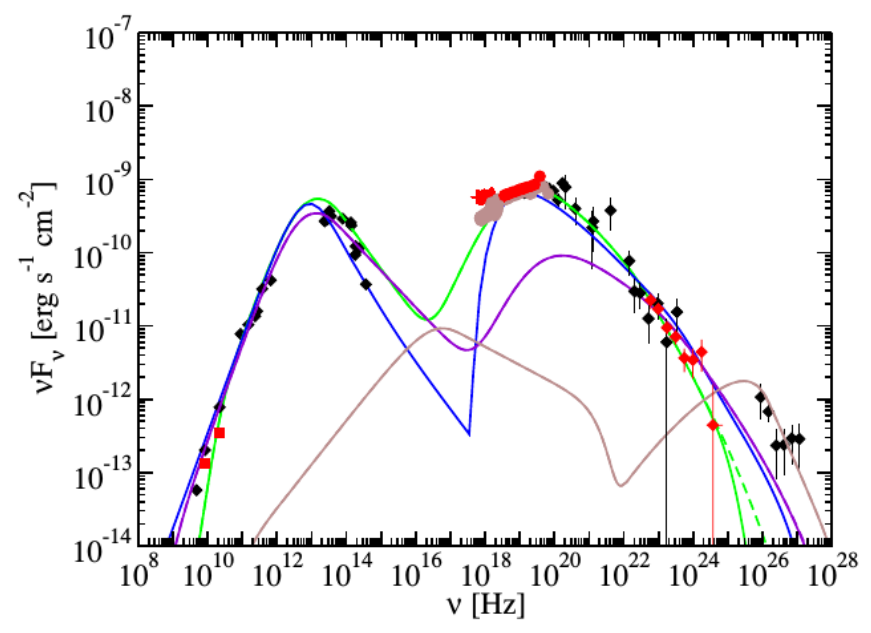

Figure 1: The broad band SED of the core of Cen A depicting numerous observations with model fits described in [10]. The symbols are observational data points: these are VLBI (red squares), Swift-XRT (red crosses), Suzaku X-rays (brown circles), Swift-BAT (red circles), Fermi-LAT (red diamonds), H.E.S.S. and other archival data (black symbols). The green model fits synchrotron self-Compton emission, while removing $\gamma \gamma$ absorption from the latter produces the dashed green curve. The violet curve is a fit that attempts to account for the highest energy gamma rays, while the brown curve attempts to fit the H.E.S.S. data. Considering the possibility of a decelerating relativistic outflow [11] produces the blue fit.

The data points in Figure 1 range from radio (at the lowest energy regime) to the VHE $\gamma$-rays at the highest energy part. The red circles at the peak of the second hump show the X-ray data from Swift-XRT ${ }^{1}$. The red diamonds depicting the HE $\gamma$-rays are obtained from the Fermi-LAT. As can be seen in Figure 1, various models successfully reproduce and fit the large part of the observed multi-wavelength data, characterizing the SED of Cen A. The first peak is generally associated with synchrotron emission from relativistic electrons, while second peak in the HE regime is explained using leptonic and hadronic models. In the one-zone leptonic case (shown in green in the SED), $\gamma$-rays are produced by the upscattering of low energy photons by the relativistic electrons through the inverse-Compton process by a single emission region (typically a spherical blob) traveling at relativistic speed along the jet. These target low energy photon populations can be internal or external to the jet system. Such a single-zone SSC (also used for various blazars) is usually popular to describe this component. Also shown by others, e.g. [12] and also evident from Figure 1, one can invoke a second emission zone (here shown in brown) with differing input parameters to account for the VHE data points. These two-zone scenario has been recently employed by the Fermi-LAT and H.E.S.S. collaborations to fully explain the entire $\gamma$-ray of the SED of Cen A.

Alternatively (for AGN in general), the hadronic explanation of the HE peak uses energetic protons (interacting with low-energy photons) that produce $\gamma$-rays after photo-pion production that leads to

\footnotetext{
${ }^{1}$ Swift-XRT is the X-Ray Telescope also on board Swift for measuring the fluxes, spectra, and light curves of GRBs sensitive in the $0.2-10 \mathrm{keV}$ band.
} 
pion and muon decay processes (or from proto-synchrotron radiation). These hadronic models will typically require an input of rather large power in the proton population (see e.g. [13]) reaching $10^{47}-10^{49} \mathrm{erg} \cdot \mathrm{s}^{-1}$. The corresponding luminosity estimates are much lower, by even 2 or more orders of magnitude for leptonic models - this essentially attributed to electrons radiating more efficiently.

A combination of leptonic and hadronic (leptohadronic) models have also been used in successful reproduction of the $\mathrm{HE}$ tail of the SED of Cen $\mathrm{A}$ where the $\mathrm{TeV}$ component is considered as an emission from relativistic proton population that interacts with the photons (photohadronic interaction) produced by the primary leptonic component [12]. Another nearby radio galaxy, IC 310 (which features a double-peak structure up to a few $\mathrm{GeV}$ ) is another example where photohadronic interactions explains the observed $\mathrm{GeV}$ through $\mathrm{TeV}$ spectra [14].

It could be that $\gamma \gamma$-absorption might give an indication of this component, and indeed $\gamma \gamma$ absorption in the BLR of blazars has been considered by several authors (see e.g. [15, 1, 2]) who reported that significant $\gamma \gamma$ absorption by the BLR can be avoided by having the $\gamma$-ray emission region outside the BLR.

\section{Multi-wavelength Observations}

To study the long-term activity of Cen A, we obtained contemporary data spanning about 6.8 years of hard X-rays and high-energy $\gamma$-rays. In addition to these, VHE $\gamma$-ray emission was probed for any variability.

The main factor that drove the specific data selection was the pressing need of contemporaneous data with the H.E.S.S. (High-Energy Stereoscopic System) observatory [16, 17]. The next subsections describe these observations briefly.

\subsection{VHE $\gamma$-ray Data}

Long-term $\mathrm{TeV} \gamma$-ray data were obtained from H.E.S.S., one of the current ground-based Imaging Atmospheric Cherenkov Telescopes (IACTs). These data spanned the period from 2004 till 2011 the period in which the system operated as H.E.S.S. Phase I. In these phase, the system with four $12 \mathrm{~m}$ IACTs (each of $108 \mathrm{~m}^{2}$ mirror area) arranged in form of a square of side-length of $120 \mathrm{~m}$. Unfortunately, these quiescent state observations of Cen $\mathrm{A}$ in the $\mathrm{TeV}$ band resulted in a rather low-significance light curve not allowing meaningful light curve correlation analysis with other wavebands.

No data where included from the H.E.S.S. Phase II period when the system had an additional $28 \mathrm{~m}$ telescope (with mirror area amounting to $614 \mathrm{~m}^{2}$ ) added.

\subsection{X-ray Data}

Hard X-ray data $(15-150 \mathrm{keV})$ from the Burst Alert Telescope (BAT)[18] on board the Swift observatory was used to obtain a 6.8 year span light curve. While primarily designed for gamma- 
ray burst (GRB) detection and rapid triggering, BAT is an efficient hard X-ray monitor instrument [19], releasing "Swift/BAT transient monitor results provided by the Swift/BAT team" [20]. These data are readily available from the BAT transient source online repository ${ }^{2}$, and have been used to produce the light curve binned in 14 day bins in the period from MJD 54703.6 to MJD 57195.6 (i.e. between 8 August, 2008 and 22 June, 2015). The starting date of this time window is set by the mission operation date of the Fermi-LAT, and the ending date by the time of analyses.

\subsection{HE Gamma-ray Data}

High-energy $\gamma$-ray $(100 \mathrm{MeV}-500 \mathrm{GeV})$ observations of Cen A that were contemporaneous to the Swift-BAT data, from the Large Area Telescope (LAT), the primary instrument on board the Fermi gamma-ray Space Telescope (Fermi) [21], were used to extract the $\gamma$-ray light curve. We employed the publicly available Fermi-LAT Pass 8 production [22], which has been optimized for point-like sources after a comprehensive review of $\gamma$-ray event analysis. Released in 2015, Pass 8 is considered to be the event reconstruction and analysis realizing the full scientific potential of the instrument by increasing the effective area, improving the point spread function, and widening the energy range that LAT is sensitive to. We performed unbinned analysis in the pylikelihood package of Science Tools (v10rOp5) to obtain the light curve in the energy range 0.1 to $500 \mathrm{GeV}$ within $10^{\circ}$ region of interest centered at the position of Cen A. In cases of small number of events per bin (e.g., when far away from the Galactic plane), LAT recommends the usage of unbinned analysis for time series data.

Assuming Cen A as having an underlying log-parabolic spectrum, we utilized P8R2_SOURCE_V6 as the instrument response functions, while gtmktime was used to select good observation time intervals of the spacecraft. The diffuse emission from our Galaxy was taken into account using the gll_iem_v06.fits model while iso_P8R2_SOURCE_V6_v06.txt was the appropriate model for background cosmic rays and diffuse extragalactic $\gamma$ rays. The flux normalization of Cen A, the Galactic and isotropic templates, and nearby bright sources were freed in the analysis.

Figure 3 shows the $\mathrm{X}$-ray and $\gamma$-ray light curves described above. The light curves span 6.8 years of observations sampled in 14 day bins.

\section{Time-lag Analysis}

\subsection{The Discrete Correlation Function}

When multiple frequency band light curves have been observed from a source for a sufficiently long time, then the natural question is if these signals are in any way correlated. This question was answered traditionally by performing some correlation function analysis. A method that avoids interpolation of the data in the process, and which also deals with spurious effects due to correlated errors, has been proposed by [23]. This time series analysis, called the discrete correlation function (DCF), is powerful in that it can even handle sparse and or unevenly sampled light curves. The DCF progressively iterates through time-lags and computes a discrete correlation coefficient for each lag.

\footnotetext{
${ }^{2}$ The publicly available monitor web page, http://swift.gsfc.nasa.gov/docs/swift/results/transients/, provides light curves for astrophysical sources observed with Swift-BAT.
} 


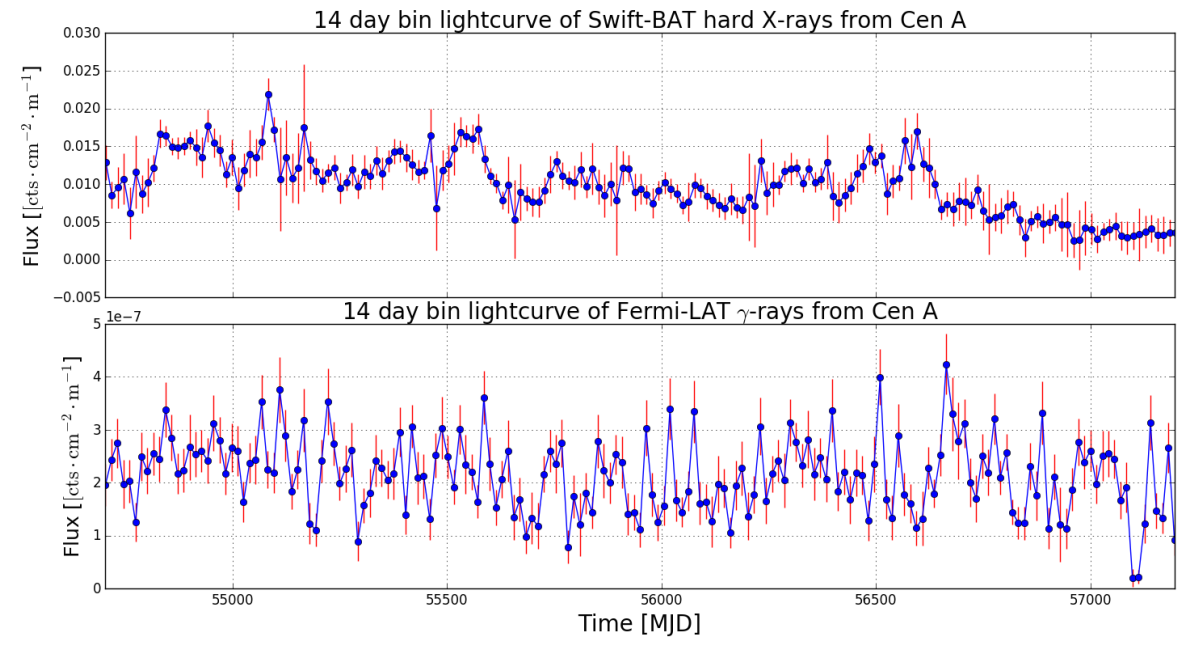

Figure 2: The Swift-BAT and Fermi-LAT light curves are shown here, where it can be clearly seen that the sampling of the X-rays in 14-day bins is much better over the Fermi-LAT binning.

This essentially means that each pair of points (one from each of the actual two light curves), gives one correlation value at a given lag. This enables an evaluation of the correlation between the two time series as a function of difference in arrival time of the signals. The result can be interpreted as trailing or leading of the first input signal if the lag is positive or negative, respectively.

\subsection{Monte Carlo Simulation of Light Curves}

In order to quantify the statistical significance of the DCF computed, we used the observed light curve in each band to simulate, via a Monte Carlo approach, 20000 light curves. To accomplish this, an algorithm proposed by [24] and later improved by [25], was used. A Python implementation of this approach is published by [26].

For each observed light curve (the HE $\gamma$-ray and X-ray curves), we used the algorithm by [26] to extract the underlying power spectral densities (PSDs) and probability density functions (PDFs). These PSDs and PDFs are then used, also as implemented by [26], to simulate light curves that resemble the observations. In this way, we simulated two pairs of 20000 light curves resembling the $\gamma$-ray and the X-rays, respectively. We then obtained correlation coefficients (using the [23] framework) by correlating each of the 20000 simulated light curves against the other observed light curve. The simulated light curves feature the same variability and statistical properties as the observed light curves. These 20000 simulated products were iteratively cross-correlated against the other observed waveband. For a given time lag, we sorted the correlation coefficients and determine the upper and lower $5 \%$ of these, from which we establish contour lines defined by the two outer boundaries containing $95 \%$ of the coefficients. These limits derived from the contour boundaries as the $95 \%$ confidence level of the DCF process is used as a statistical significance check of the correlations. 


\subsection{DCF Computation Result}

Figure 3 shows the discrete correlation function between hard X-rays and HE $\gamma$ rays of Cen A. This is essentially a plot of the correlation coefficient as a function of time-lag where the correlation was performed as described in Section 4.1. This distribution is approximately Gaussian for which the width is a measure of the uncertainty in the time lag. Overlaid on the correlation, is a band bounded by the $95 \%$ confidence level (see Section 4.2) which is surpassed by the peak of the correlation function. It can be noticed that the result hints to a scenario where the X-rays observed from the Swift-BAT instrument leads the Fermi-LAT $\gamma$-rays by 25 days (see the peak of the Gaussian fit which has a standard deviation of 152). The correlation coefficient at the peak is $0.35 \pm 0.14$. The result is established to have achieved over $95 \%$ confidence level using Monte Carlo simulations of light curves as described in Section 4.2.

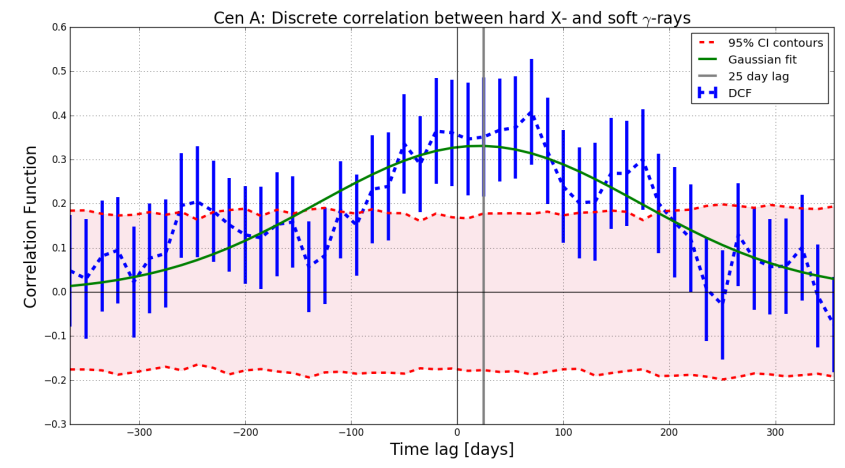

Figure 3: DCF result: The dotted blue curve denotes the DCF as a function of time-lag (with a overlaid green Gaussian fit), while the error bars on the correlation coefficients are propagated from the errors on the flux measurements. The peak at 25.0 day time lag (indicated by the solid gray vertical line), is the deduced time-lag by which HE $\gamma$-rays trail behind the X-rays. The reddish band is bounded by the $95 \%$ confidence contours. The lag bin width of the shown DCF is 15 days, constraint by the observation sampling rate and the duration of the observations. The latter also serves as a boundary to the lag range tested. This presented choice of the lag bin width and its range were arrived at by iterative visual inspection through these intervals.

\section{Summary \& Discussion}

The light curve correlation between the 6.8 years of HE gamma-ray and the X-ray data of Cen A considered here, resulted in a hint of a possible time-lag of about 25 days of the gamma-ray emission behind the X-rays. The discrete correlation function (DCF) associated with this lag peaked at the value of $0.35 \pm 0.14$. Although the time-lag is a mere hint, the correlation is statistically significant beyond the $95 \%$ confidence level from Monte Carlo simulations of the light curves.

In the event that further studies confirms a time-lag of this magnitude, then we can couple that to arguments of light-travel time, to set a size constraint on the BLR of the system through

$$
\begin{aligned}
\Delta R & \sim c \Delta t_{\mathrm{lag}} \\
& \sim 6.5 \times 10^{16} \mathrm{~cm}
\end{aligned}
$$


This would then indicate that the size of the BLR near the central engine is about 0.02 parsec. This estimate has considered the fact that we neglect relativistic beaming for a typical radio galaxy for which the jet is largely misaligned to our line of sight.

\section{Acknowledgements}

This project is sponsored by the German Academic Exchange Service (DAAD) (German: Deutscher Akademischer Austauschdienst), the National Research Foundation ${ }^{3}$ (NRF) and the Department of Science and Technology of the Republic of South Africa through the South African Research Chair Initiative under SARChI grant 64798, as well as the Department of Physics of the University of Namibia.

\section{References}

[1] Roustazadeh, P. \& Boettcher, M., Compton and Synchrotron Emission from Pair Cascades in Radio Galaxies, AAS/High Energy Astrophysics Division, 12, 4.08, (2011).

[2] Böttcher, M. \& Els, P., Gamma-Gamma Absorption in the Broad Line Region Radiation Fields of Gamma-Ray Blazars, ApJ, 821, 102-106, 2016.

[3] Fabian, A. C., Rees, M. J., Stella, L. \& White, N. E., X-ray fluorescence from the inner disc in Cygnus $X-1$, MNRAS, 238, 729-736, (1989).

[4] Israel, F. P., Centaurus A - NGC 5128, Astronomy \& Astrophysics Review, 8, 237-278, (1998).

[5] Marconi, A., Pastorini, G., Pacini, F., Axon, D. J., Capetti, A., Macchetto, D., Koekemoer, A. M. \& Schreier, E. J., The supermassive black hole in Centaurus A: a benchmark for gas kinematical measurements, AAP, 448, 921-953, (2006).

[6] Häring-Neumayer, N., Cappellari, M., Rix, H.-W., Hartung, M., Prieto, M. A., Meisenheimer, K. \& Lenzen, R., VLT Diffraction-limited Imaging and Spectroscopy in the NIR: Weighing the Black Hole in Centaurus A with NACO, ApJ, 643, 226-237, (2006).

[7] Fanaroff, B. L. \& Riley, J. M., The morphology of extragalactic radio sources of high and low luminosity, MNRAS, 167, 31P-36P, (1974).

[8] Graham, J. A., Brown, A. M., Boehm, C., Lacroix, T., Chadwick, P. M., \& Joseph, S., The Fermi-LAT spectrum of Centaurus A - Analysis and interpretations, AIP Conference Proceedings, 1792, 050004, (2017).

[9] Abdalla, H., Abramowski, A. \& Aharonian, F. et al. (for the H. E. S. S. Collaboration), The $\gamma$-ray spectrum of the core of Centaurus A as observed with H.E.S.S. and Fermi-LAT, ArXiv e-prints, 1807.07375, (2018).

[10] Abdo, A. A, Ackermann, M., \& Ajello, M. et al. (for the Fermi-LAT Collaboration), Fermi Large Area Telescope View of the Core of the Radio Galaxy Centaurus A, ApJ, 719, 1433-1444, (2010).

[11] Georganopoulos, M, \& Kazanas, D., Decelerating Flows in TeV Blazars: A Resolution to the BL Lacertae-FR I Unification Problem, ApJL, 594, L27 (2003).

\footnotetext{
${ }^{3}$ Disclaimer: Any opinion, finding and conclusion or recommendation expressed in this material is that of the authors and the NRF does not accept any liability in this regard.
} 
[12] Petropoulou, M., Lefa, E., Dimitrakoudis, S. \& Mastichiadis, A., One-zone synchrotron self-Compton model for the core emission of Centaurus A revisited, AAP, 562, A12, (2014).

[13] Böttcher, M., Reimer, A., Sweeney, K. \& Prakash, A., Leptonic and Hadronic Modeling of Fermi-detected Blazars, ApJ, 768, 54, (2013).

[14] Fraija, N., Marinelli, A., Galván-Gámez, A. \& Aguilar-Ruiz, E., Modeling the spectral energy distribution of the radio galaxy IC310, Astroparticle Physics, 89, 14-22, (2017).

[15] Protheroe, R. J. \& Biermann, P. L., Photon-photon absorption above a molecular cloud torus in blazars, Astropart. Physics, 6, 293-300, (1997).

[16] Bernlöhr, K., Carrol, O., Cornils, R., Elfahem, S., Espigat, P., Gillessen, S., Heinzelmann, G., Hermann, G., Hofmann, W., Horns, D., Jung, I., Kankanyan, R., Katona, A., Khelifi, B.,

Krawczynski, H., Panter, M., Punch, M., Rayner, S., Rowell, G., Tluczykont, M. \& van Staa, R., The optical system of the H.E.S.S. imaging atmospheric Cherenkov telescopes. Part I: layout and components of the system, Astropart. Physics, 20, 111-128, (2003).

[17] Cornils, R., Gillessen, S., Jung, I., Hofmann, W., Beilicke, M., Bernlöhr, K., Carrol, O., Elfahem, S., Heinzelmann, G., Hermann, G., Horns, D., Kankanyan, R., Katona, A., Krawczynski, H., Panter, M., Rayner, S., Rowell, G., Tluczykont, M. \& van Staa, R., The optical system of the H.E.S.S. imaging atmospheric Cherenkov telescopes. Part II: mirror alignment and point spread function, Astropart. Physics, 20, 129-143, (2003).

[18] Gehrels, N., Chincarini, G., \& Giommi, K.O., et al (for the Swift Sciebce Team) The Swift Gamma-Ray Burst Mission, ApJ, 611, 1005-1020, (2004).

[19] Krimm, H. A., Barthelmy, S. D., Markwardt, C. B., Sanwal, D., Tueller, J., Gehrels, N. \& Swift/BAT Team, The Swift-BAT Hard X-ray Transient Monitor, Bulletin of the American Astronomical Society, 38, 374, (2006).

[20] Krimm, H. A., Holland, S. T., Corbet, R. H. D., Pearlman, A. B., Romano, P., Kennea, J. A., Bloom, J. S., Barthelmy, S. D., Baumgartner, W. H., Cummings, J. R., Gehrels, N., Lien, A. Y., Markwardt, C. B., Palmer, D. M., Sakamoto, T., Stamatikos, M. \& Ukwatta, T. N., The Swift/BAT Hard X-Ray Transient Monitor, ApJSS, 209, 14, (2013).

[21] Atwood, W. B., Abdo, A. A., \& Ackermann, et al., The Large Area Telescope on the Fermi Gamma-Ray Space Telescope Mission, ApJ, 697, 1071-1102, (2009).

[22] Atwood, W., Albert, A., Baldini, L., Tinivella, M., Bregeon, J., Pesce-Rollins, M., Sgrò, C., Bruel, P., Charles, E., Drlica-Wagner, A., Franckowiak, A., Jogler, T., Rochester, L., Usher, T., Wood, M., Cohen-Tanugi, J. \& Zimmer, S. (for the Fermi-LAT Collaboration), Pass 8: Toward the Full Realization of the Fermi-LAT Scientific Potential, ArXiv e-prints 1303.3514, (2013).

[23] Edelson, R. A. \& Krolik, J. H., The discrete correlation function: A new method for analyzing unevenly sampled variability data, ESA Special Publication, 281, 646-659,(June 1988)

[24] Timmer, J. \& Koenig, M., On generating power law noise, AAP, 300, 707, (1995).

[25] Emmanoulopoulos, D., McHardy, I. M. \& Papadakis, I. E., Generating artificial light curves: revisited and updated, MNRAS, 433, 907-927, (2013).

[26] Connolly, S., A Python Code for the Emmanoulopoulos et al. [arXiv:1305.0304] Light Curve Simulation Algorithm, ArXiv e-print 1503.06676, (2015). 